\title{
Modelling the radiation action for the estimation of biological effects in humans
}

\author{
F. Ballarini ${ }^{1,2, a}$, M.V. Garzelli ${ }^{2,3}$, G. Givone ${ }^{1,2}$, A. Mairani ${ }^{1,2}$, A. Ottolenghi ${ }^{1,2}$, D. Scannicchio ${ }^{1,2}$, S. Trovati ${ }^{1,2,4}$, and A. Zanini ${ }^{2}$ \\ 1 University of Pavia, Nuclear and Theoretical Physics Department, via Bassi 6, 27100 Pavia, Italy \\ 2 INFN (National Institute of Nuclear Physics), Italy \\ 3 University of Milano, Physics Department, via Celoria 16, 20133 Milano, Italy \\ 4 CERN, Geneva, Switzerland
}

\begin{abstract}
It is well known that ionizing radiation can induce biological effects at different levels, from DNA, chromosomes and cells up to tissues, organs and entire organisms. Theoretical models and Monte Carlo codes, especially those based on radiation track structure, can be of great help to elucidate the underlying mechanisms and to perform reliable predictions where data are lacking. In this work we will present and discuss a mechanistic $a b$ initio model and a Monte Carlo code able to simulate the induction of chromosome aberrations (CAs) in human cells. This endpoint is particularly relevant, since some aberration types can lead to cell death, while others can lead to cell conversion to malignancy. The model is based on the hypothesis that only clustered lesions (CLs) of the DNA double-helix can evolve into aberrations. Simulated dose-response curves for CAs induced by different radiation types (including heavy ions) will be shown, together with applications to cancer risk estimation and biodosimetry. In this framework, we will also discuss examples of medical applications - including astronauts' exposure to space radiation - obtained with the FLUKA code, also taking into account the role of nuclear interactions.
\end{abstract}

\section{Introduction}

Human beings can be exposed to ionizing radiation from different sources such as the Earth natural background, medical diagnostics or treatments and occupational exposure. However, the involved dose and dose rates can be very different: the total Earth background is about $2-3 \mathrm{mSv} / \mathrm{yr}$, whereas for example an abdomen CT requires doses of the order of $10 \mathrm{mSv}$. Tumor treatments involve much higher doses (about 50 Gy in "standard" treatments, which generally consist of 20-25 fractions). However, it has to be taken into account that it's only the tumor which receives such high doses, though also normal tissues surrounding the tumor volume can receive (unwanted) small doses.

Both for the Earth background and for medical exposure, low-LET (Linear Energy Transfer) radiation, typically $\mathrm{X}$ rays, is involved. However, it is worth mentioning that an increasing number of radiotherapy facilities are now making use of protons or heavier ions, typically Carbon [1]. The latter are currently used at NIRS in Chiba, Japan, and at GSI in Darmstadt, Germany. New Carbon facilities are under construction in Pavia, Italy, in Heidelberg, Germany, and in other Japanese locations. Both protons and heavier ions are characterized by a localization of energy deposition in the so-called "Bragg peak" region, that can allow for an increase of Tumor Control Probability (TCP), particularly for certain radioresistant tumors, with low Normal Tissue Complication Probability (NTCP). While ions such as Argon have shown unacceptable complications to normal tissues, Carbon beams are particularly suitable because their Relative Biological Effectiveness is sufficiently low (approximately 1, as for protons) in the plateau before the Bragg peak, whereas it is

\footnotetext{
${ }^{a}$ Present author, e-mail: francesca.ballarini@pv.infn.it
}

higher (up to 3-4 and even more, to be compared with the typical 1.1 value of proton beams) in the region of the peak.

Another scenario where high LET radiation is involved is the exposure of astronauts to space radiation. The spectrum of Galactic Cosmic Rays, to which astronauts are exposed continuously, consists of about $87 \%$ protons, $12 \% \mathrm{He}$ ions and $1 \%$ heavier ions in fluence. Although the dose rate is relatively low (of the order of $1 \mathrm{mSv} /$ day in free space), radiation can represent a serious threat for the crewmembers of longterm missions. This is especially true for missions in deep space outside the Geomagnetic field, that is the case of lunar missions and possible travels to Mars. The exposure scenario in space is further complicated by "Solar Particle Events" (SPEs), which are occasional but almost unpredictable injections of high fluxes (up to more than $10^{10}$ particles $\cdot \mathrm{cm}^{-2}$ in few hours, to be compared with 4 particles $\cdot \mathrm{cm}^{-2} \cdot \mathrm{s}^{-1}$ for GCR) of charged particles coming from the Sun, mainly protons with energies below a few hundred $\mathrm{MeV}$. In this context, after a general overview on the effects of ionizing radiation on biological targets (sect. 2), in section 3 we will present examples of calculations related to the characterization of therapeutic proton beams and the estimation of space radiation doses for astronauts.

To characterize the action of ionizing radiation at the organ/organsm level, it is necessary to understand the mechanisms underlying radiobiological damage at cellular and subcellular level. Therefore, in section 4 we will focus on the mechanisms underlying the induction of chromosome aberrations (CAs), which are a particularly relevant endpoint because they are correlated both with cell death and with cell conversion to malignancy, implying that CAs can influence the "fate" of the cells. More specifically, we will present a mechanistic model and Monte Carlo code able to simulate the induction of the main aberration types following exposure of 
human cells to different radiation types including ions such as Carbon and Iron, which are of interest for hadrontherapy and space radiation protection, respectively.

\section{A brief overview on the effects of ionizing radiation on biological targets}

The action of ionizing radiation in biological targets is a stochastic, multi-step process involving several orders of magnitude, both in the space scale and in the time scale. Primary energy deposition, which can be considered completed at $10^{-15}$ seconds after irradiation ("physical stage"), produces a spatial distribution of ionized and excited molecules. Although the DNA occupies only $\approx 2 \%$ of the cell nucleus volume, it is the most important target for ionizing radiation. Also energy deposition in the surrounding water plays a significant role, since during the so-called "prechemical stage" (from $10^{-15}$ to $10^{-12} \mathrm{~s}$ after irradiation) the dissociation of ionized and excited $\mathrm{H}_{2} \mathrm{O}$ molecules gives rise to free radicals (e.g., the $\mathrm{OH}$ radical) that can attack the double helix constituents and produce DNA damage.

DNA damage, either produced by direct energy deposition in the double-helix atoms ("direct damage") or produced by free radical attack ("indirect damage"), is processed by specific repair enzymes. The oxygen level in the environment is an important parameter, since $\mathrm{O}_{2}$ can link damaged sites preventing repair and thus acting as a "damage fixer". This has implications for radiation therapy, since hypoxic tumors result to be particularly radio-resistant. In case of incorrect DNA repair, the initial damage can evolve within a few hours into endpoints such as gene mutations and chromosome aberrations, which in turn may lead to cell death, or cell conversion to malignancy and possibly cancer. While cell death and conversion to malignancy can be detected in vitro after a few days or weeks, cancer is characterized by long latency times of the order of several years.

To have a quantitative idea of the yields of the various damage types, one may consider that a mammalian cell nucleus irradiated with 1 Gy of gamma rays will be affected, on average, by about 100,000 ionizations (2,000 in the DNA), 1,000 DNA single-strand breaks (ssb), 40 doublestrand breaks (dsb), 1 chromosome aberration (or even less) and $10^{-5}$ gene-specific mutations. The probability of cancer development is far less than $10^{-5}$.

Since the double-helix diameter is about 2 nanometers, the knowledge of radiation track structure at the nm level is an essential prerequisite for understanding the mechanisms underlying the processes of interest. The expression "track structure" refers to a set of information including, for each energy deposition event (typically a ionization or excitation), spatial coordinates of the event, event type and amount of deposited energy. The higher-order organization characterizing mammalian DNA is another important modulating factor. Each DNA molecule winds around globular proteins ("histones") to form structures called nucleosomes, which in turn are packed to form a $30-\mathrm{nm}$ diameter chromatin fiber. The fiber is organized as a succession of loops that, for most of the cell cycle duration, are localized within distinct domains called chromosome territories, with linear dimensions of the order of $\approx 1 \mu \mathrm{m}$. During the last decade, when the advances in confocal microscopy allowed more detailed investigation of nuclear architecture, it became clear that DNA damage processing mainly occurs in small channels separating neighbouring domains. Therefore, when dealing with chromosome aberration induction, the spatial distribution of the initial energy depositions needs to be considered not only at the nanometer scale (i.e., the double helix level), but also at the micrometer scale (i.e., the chromosome level).

\section{Effects at the level of organs and organism: examples of calculations of interest for radiotherapy and space radiation protection}

In previous works aimed to the characterization of therapeutic proton beams, calculations have been performed on physical and "biological" doses along the plateau and the Spread-Out Bragg Peak (SOBP) of the $72 \mathrm{MeV}$ proton beam used for the treatment of ocular tumors at the Paul Sherrer Institut (PSI) in Switzerland [2]. Such calculations have been carried out with FLUKA, a multi-purpose Monte Carlo code able to deal with transport and interaction of electromagnetic and hadronic particles over a wide energy range in any material [3-5]. The "biological dose" has been defined as the average number of "Complex Lesions" (CLs) per cell. CLs, which are clustered breaks of the DNA double helix, have been operatively defined and calculated basing on radiation trackstructure simulations at the nm level [6]. Importantly, the beam Relative Biological Effectiveness (RBE) was found to be 1.2 along most of the SOBP (except for an increase in the peak distal part), consistent with the constant value of 1.1 adopted in therapeutic practice with protons. While the absorbed dose was roughly constant with depth throughout the entire SOBP, the calculated profile of "biological" dose showed a sharp increase at the distal part of the SOBP due to the presence of protons with low energy and thus high LET and biological effectiveness. In order to quantify the role of nuclear interactions, the contributions from the various radiation field components have been calculated separately. Secondary particles produced by nuclear interaction accounted for less than $4 \%$ of the total absorbed dose in most of the SOBP, and disappeared in its distal part. The relative contribution of nuclear reaction products to the biological dose was larger with respect to the absorbed dose, reaching values of about $12 \%$ in the proximal part of the SOBP. The method described above was then extended to the characterization of a "virtual" $160 \mathrm{MeV}$ proton beam [7]. These results confirm the reliability of the RBE value of 1.1 generally adopted in proton therapy.

Concerning space radiation protection, in the framework of the FLUKA collaboration, calculations have been performed on astronaut doses following exposure to space radiation under different shielding conditions. These results have been obtained coupling FLUKA with two anthropomorphic phantoms, i.e., a mathematical model based on combinatorial geometry [8] and a "voxel" model constructed starting from whole-body CT data [9]. Both phantoms have been inserted into an Al cylindrical shell of variable thickness, i.e., 1 and 
Table 1. Organ-averaged dose equivalents (in Sv) calculated for the August 1972 SPE for different values of Al shielding thickness (in g. $\left.\mathrm{cm}^{-2}\right)$.

\begin{tabular}{cccc}
\hline Al shield & Skin & Eye & Red Bone Marrow \\
\hline 1 & 13.31 & 6.89 & 1.80 \\
2 & 7.25 & 4.90 & 1.32 \\
5 & 2.23 & 1.60 & 0.62 \\
10 & 0.62 & 0.56 & 0.25 \\
\hline
\end{tabular}

Table 2. GCR annual effective doses (in Sv) calculated for for different values of $\mathrm{Al}$ shielding thickness (in $\mathrm{g} \mathrm{cm}^{-2}$ ).

\begin{tabular}{ccc}
\hline Al shield & Male dose & Female dose \\
\hline 1 & 0.47 & 0.44 \\
2 & 0.46 & 0.41 \\
3 & 0.43 & 0.41 \\
5 & 0.42 & 0.41 \\
\hline
\end{tabular}

$2 \mathrm{~g} \cdot \mathrm{cm}^{-2}$ (nominal spacesuit and lightly shielded spacecraft), $5 \mathrm{~g} \cdot \mathrm{cm}^{-2}$ (nominal spacecraft) and $10 \mathrm{~g} \cdot \mathrm{cm}^{-2}$ (storm shelter to be used in case of SPE). Organ-averaged dose equivalent values, as well as effective doses, have been calculated according to ICRP 60 [10]. Table 1 shows organ-averaged dose equivalents (for skin, eyes and red bone marrow, which are the reference organs for radiation protection against deterministic effects) calculated by simulating exposure to the August 1972 SPE. As expected, all dose types decrease dramatically by increasing the shielding. For each considered thickness of the Al shielding, the dose due to the products of nuclear reactions of primary protons with the shield and the human body has been calculated separately. Although the role of nuclear reaction products is not negligible (especially for heavy shielding with respect to light shielding, due to target fragmentation), primary protons play a major role. According to these calculations, in case of a solar event similar to the August $1972 \mathrm{SPE}$ an Al storm shelter of $10 \mathrm{~g} / \mathrm{cm}^{2} \mathrm{Al}$ would be sufficient to respect the NCRP limits for 30-days missions in Low Earth Orbit, which are 1.5, 1.0 and 0.25 for skin, eye lenses and Blood Forming Organs, respectively [11]. However, since these limits refer to Low Earth Orbit missions within the Geomagnetic field, numerical comparisons with deep space scenarios should be taken with caution, considering such numbers as mere guidelines while waiting for the introduction of new reference values specific for deep space.

Table 2 shows annual effective doses for GCR exposure at solar minimum (in Sv). According to these results, a hypothetical 2-years mission in deep space (typical duration of a possible mission to Mars) under solar minimum conditions would allow to respect the NCRP career limits [11] for males who are at least 35-years-old (limit: $\geq 1 \mathrm{~Sv}$ ) and females of at least 45 (limit: $\geq 0.9 \mathrm{~Sv}$ ). Again, comparisons with these limits need to be considered with caution due to the reasons mentioned above.

As expected because of the GCR high energy, GCR doses do not decrease significantly by increasing the Al shielding. As for the August 1972 SPE, for each Al thickness value the contributions from primary ions and nuclear interaction products have been calculated separately. Similarly to what obtained for SPEs, the contribution of nuclear reaction
Table 3. Calculated GCR doses (in mGy/year) in a water phantom placed inside a cylindrical habitat under 3 metres regolith.

\begin{tabular}{cc}
\hline Total dose & 7.2 \\
\hline Primary ions & 0.0 \\
Secondary particles & 7.2 \\
neutrons & 6.7 \\
\hline
\end{tabular}

products was found to increase with the Al shield thickness. However, in comparison to SPEs, in the case of GCR the relative contribution of nuclear interaction products was much higher, being roughly the same as that of the primary ions.

Following GCR dose calculations in deep space, FLUKA has been recently applied to analogous calculations for the Moon [12]. More specifically, a cylindrical habitat was simulated, placed in a cavity under the Moon surface. Outside the habitat, the cavity was filled with regolith, and the habitat itself was covered with a 3 metres thick regolith layer. A water phantom was placed in the habitat.

Preliminary calculations for GCR irradiation showed that the total dose in the phantom was $7.2 \mathrm{mGy} /$ year. As expected, this dose was entirely due to secondary particles (including neutrons). Interestingly, neutrons alone delivered a dose of $6.7 \mathrm{mGy} /$ year, as reported in table 3 .

The cosmic ray simulation tools are courtesy of the FLUKA Collaboration [3-5, 13, 14].

\section{Induction of chromosome aberrations by ionizing radiation}

It is now widely accepted that chromosome aberrations are due to breakage of the DNA double helix and subsequent incorrect rejoining of the involved DNA fragments. However, various aspects of the underlying mechanisms - such as the role of clustered DNA damage and of chromosome organization within the cell nucleus - need further clarification. In this framework, theoretical models and Monte Carlo codes can be of great help to clarify the mechanisms leading to initial energy deposition by radiation to the formation of chromosome aberrations.

The main assumption of our model [15-17] consists of considering chromosome aberrations as the "evolution" of clustered DNA breaks or "Complex Lesions" (CLs), which have been operationally defined as "at least two breaks in each of the two DNA strands within 30 base-pairs" [6]. Regardless of the definition, which is necessary to get quantitative simulation results, the key point is that these lesions are clustered, and thus severe and difficult to be processed by the cell repair machinery.

This assumption also relies on the fact that the dependence of CLs on radiation quality reflects that shown by data on the induction of gene mutations and cell death, whereas non-clustered double-strand breaks show a much weaker dependence on the radiation type and energy. To model CA induction, each CL is assumed to produce two independent chromosome free ends. Only free ends induced in neighbouring chromosomes or in the same chromosome are allowed to join and give rise to aberrations, reflecting the experimental 


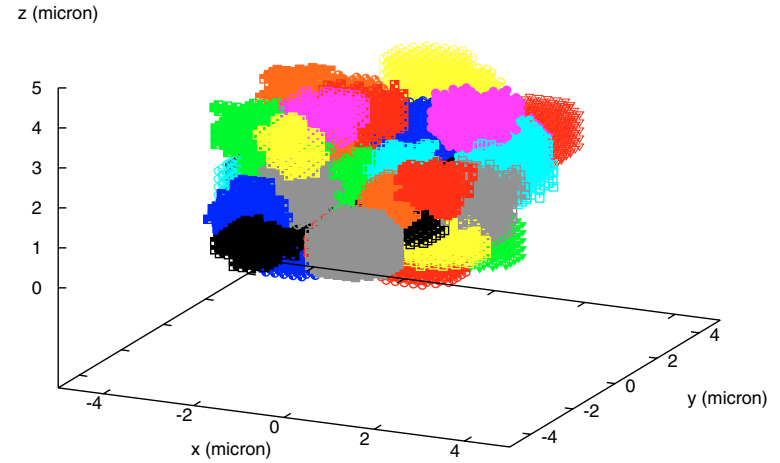

Fig. 1. Preliminary example of simulated human fibroblast cell nucleus with its 46 chromosome territories (see the text for further details).

evidence that DNA repair takes place within the channels separating the various chromosome "territories", which are basically non-overlapping intra-nuclear regions occupied by a single chromosome.

The current version of the model deals with human lymphocyte nuclei, which are modelled as $3-\mu \mathrm{m}$ radius spheres; the implementation of human fibroblast nuclei is in progress, and a preliminary example is shown in figure 1. Both for lymphocytes and for fibroblasts, the 46 chromosome territories are described as (irregular) intra-nuclear domains with volume proportional to the chromosome DNA content. Each territory consists of the union of small adjacent cubes. Repetition of chromosome territory construction with different chromosome positions provides different configurations for cell nuclei in the $\mathrm{G}_{0}$ phase of the cell cycle.

The yield (i.e., average number, taken from "event-byevent" radiation track structure simulations at the nm level) of induced $\mathrm{CL} \cdot \mathrm{Gy}^{-1} \cdot$ cell $^{-1}$ is the starting point for dose-response simulations. While for photons the lesions are randomly distributed in the cell nucleus, for light ions they are located along straight lines representing the cell nucleus traversals.

For a given dose D (in Gy), the average number of cell nucleus traversals $\mathrm{n}$ is calculated by $\mathrm{n}=\mathrm{D} \pi \mathrm{r}^{2} /(0.16 \mathrm{LET})$, where the LET is expressed in $\mathrm{keV} / \mu \mathrm{m}, \mathrm{r}$ (in $\mu \mathrm{m}$ ) is the cell nucleus radius and 0.16 is a factor coming from the conversion between Joules and eV. An actual number is extracted from a Poisson distribution. For each cell nucleus traversal, a random extraction of the point where the particle enters the nucleus provides the traversal length, being the direction fixed (irradiation with parallel beam).

The average number of CLs per unit length along a cell nucleus traversal is $\mathrm{CL} / \mu \mathrm{m}=0.16 \cdot \mathrm{CL} \mathrm{Gy}^{-1} \cdot$ cell $^{-1} \cdot \mathrm{LET}$. $\mathrm{V}^{-1}$, where $\mathrm{V}$ is the cell nucleus volume in $\mu \mathrm{m}^{3}$. For each nucleus traversal, a Poisson distribution provides an actual number of lesions. The comparison of the CL positions with the positions of the (centres of the) boxes constituting each chromosome territory allows the association of each lesion to a chromosome.

Specific background (i.e., prior to irradiation) yields for different aberration types - typically 0.001 dicentrics/cell and 0.005 translocations/cell - can be included. Both Giemsa staining (all chromosomes painted with the same colour) and FISH selective painting can be simulated; small fragments,
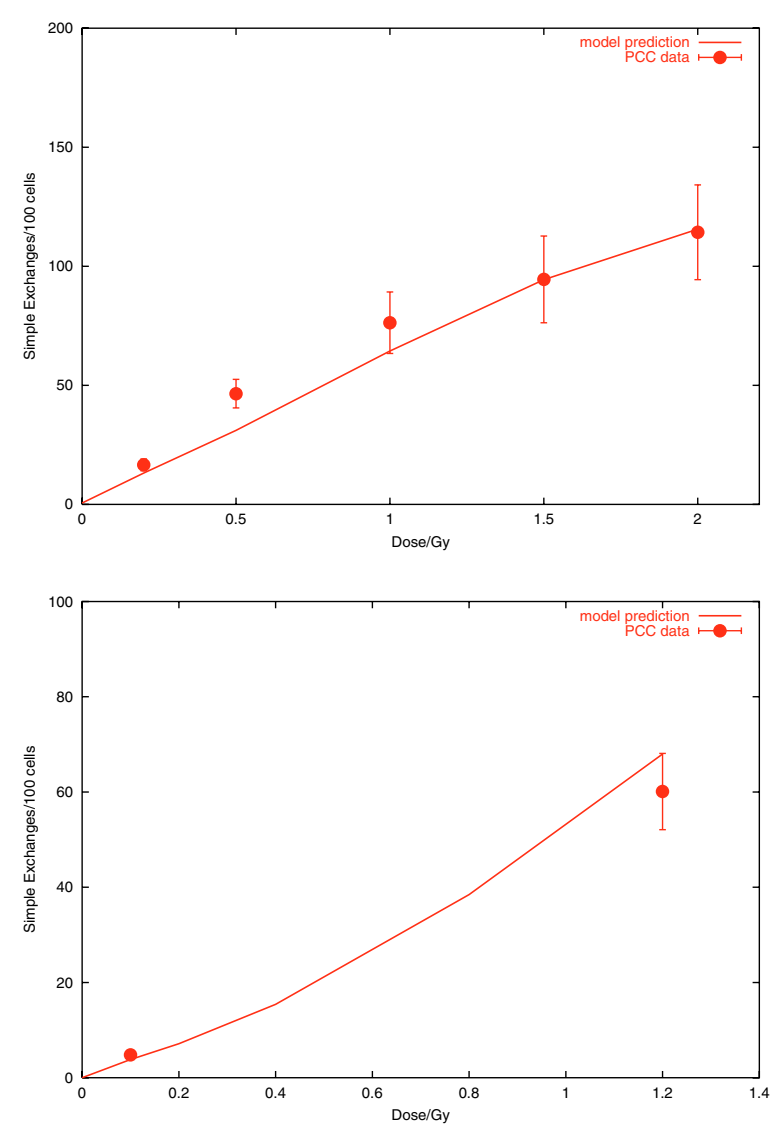

Fig. 2. Dose-response for simple exchanges (average no. per 100 cells) induced by $\mathrm{Fe}$ ions (upper panel) and $\mathrm{C}$ ions (lower panel). The lines are model predictions, whereas the points are experimental data taken from the literature [19].

i.e., with size of about $10 \mathrm{Mbp}$ (Mega base-pairs) or less, are generally not scored since they can hardly be detected in experiments. The simulation of CL induction and rejoining for a sufficiently high number of times provides statistically significant aberration yields. The repetition of the process for different dose values allows to obtain dose-response curves for the main aberration types, directly comparable with experimental data.

In previous work the model has been tested for gamma rays, protons and $\mathrm{He}$ ions by comparing simulated doseresponse curves with experimental data available in the literature, without performing any fit a posteriori. The good agreement between model predictions and experimental data for the induction of different aberration types allowed for model validation regarding both the adopted assumptions and the simulation techniques. Furthermore, the model has been applied to the induction of Chronic Myeloid Leukaemia [18] and to the estimation of chromosome aberrations observed in lymphocytes of astronauts following long-term missions onboard the Mir station and the International Space Station, on the basis of simulated gamma-ray dose responses weighted by the space radiation quality factor [17].

The extension of the model to heavy ions is in progress: as a first approach, a fraction of the DNA lesions induced by a heavy ion are "shifted" radially to model the effects of 
the so-called "delta rays", which play a significant role in determining the features of heavy-particle tracks.

An example of preliminary results is reported in figure 2, which shows dose response curves for simple exchanges (i.e. dicentrics plus translocations) induced by $1 \mathrm{GeV} / \mathrm{n}$ $\mathrm{Fe}$ ions (LET $=147 \mathrm{keV} /$ micron) and $290 \mathrm{Mev} / \mathrm{n} \mathrm{C}$ ions $(\mathrm{LET}=13.3 \mathrm{keV} /$ micron $)$. The lines represent model predictions, whereas the points are experimental data taken from the literature [19].

It is of interest for low-dose applications to report that, according to our simulations, while a single cell nucleus traversal by a $1 \mathrm{GeV} / \mathrm{n}$ proton or $\mathrm{He}$ ion resulted not to give rise to aberration yields higher than the background levels (due to their high velocity and low charge, and thus low LET), a single $1 \mathrm{GeV} / \mathrm{n}$ Iron traversal was found to induce chromosome aberration yields of the order of $0.26 /$ cell both for dicentrics and translocations.

Also in progress is the implementation of chromosome aberration transmission to the cell progeny and, on the other side, of cell killing, basing on the fact that cells carrying specific aberrations (typically dicentrics) have a significant probability of failing to duplicate.

\section{Conclusions}

In the present work, an overview was given about where humans can be exposed to radiation and about the main effects of radiation on biological targets. Some examples were reported on radiation effects at organ/organism level, i.e., on the characterization of therapeutic proton beams and the calculation of astronauts doses.

Concerning the radiation effects at cellular level, a model and a Monte Carlo code was presented, able to simulate chromosome aberration induction by different radiation types. The model is based on the assumption that clustered DNA breaks play a fundamental role in the processes leading from energy deposition to the formation of chromosome aberrations. The good agreement of the model with experimental data provided a validation of both the assumptions and the simulation techniques. Furthermore, applications of the model to biodosimetry and cancer induction were shown.

Together, these results outline the importance of nuclear data need not only for hadrontherapy and space research, but, more generally, for all those scenarios which imply exposure to mixed fields.

This work was partially supported by the European Community (EC Integrated Projects "RISCRAD" and "NOTE") and by the Italian Space Agency (ASI contract "Mo-Ma").

\section{References}

1. G. Kraft, Radiobiological effects of highly charged ions, in The Physics of Highly and Multiply Charged Ions, edited by F. Currel (Kluwer Academic, 2002).

2. M. Biaggi, F. Ballarini, W. Burkard, E. Egger, A. Ferrari, A. Ottolenghi, Nucl. Instrum. Meth. Phys. Res. B 159, 89 (1999).

3. FLUKA (Copyright 1989-2007, CERN and INFN) is a joint project of CERN and INFN; http://www.fluka.org.

4. A. Ferrari, P.R. Sala, A. Fassò, J. Ranft, FLUKA: a multi-particle transport code, CERN-2005-10 (2005), INFN/TC_05/11, SLACR-773.

5. G. Battistoni, M. Brugger, F. Cerutti, A. Fassò, A. Ferrari, E. Gadioli, S. Muraro, M. Pelliccioni, J. Ranft, S. Roesler, P.R. Sala, V. Vlachoudis, Overview of the FLUKA code and examples of use for radiation detectors, Invited talk at the Workshop on Use of Monte Carlo Techniques for Design and Analysis of Radiation Detectors, Coimbra, Sept. 2006 (Proceedings in press).

6. A. Ottolenghi, M. Merzagora, L. Tallone, M. Durante, H. Paretzke, W. Wilson, Radiat. Environ. Biophys. 34, 239 (1995).

7. M. Biaggi, F. Ballarini, W. Burkard, E. Egger, A. Ferrari, A. Ottolenghi, D. Scannicchio, Phys. Med. 17, 63 (2001).

8. M. Pelliccioni, M. Pillon, Radiat. Protec. Dosim. 67, 253 (1996).

9. M. Zankl, A. Wittmann, Radiat. Environ. Biophys 40, 153 (2001).

10. International Commission on Radiological Protection, Report No. 60 (Pergamon Press, New York, 1991).

11. National Council on Radiation Protection and Measurements, Report No. 132, Bethesda, 2000.

12. G. Givone, Laurea Thesis, University of Torino, 2006.

13. G. Battistoni, A. Ferrari, T. Montaruli, P.R. Sala, Comparison of the FLUKA calculations with CAPRICE94 data on muons in atmosphere, Astroparticle Phys. 17, 477 (2002).

14. G. Battistoni, A. Ferrari, P.R. Sala, Calculation of secondary particle in atmosphere and hadronic interactions, International J. Modern Phys. A 17, 1743 (2002).

15. F. Ballarini, A. Ottolenghi, Chromosome aberrations as biomarkers of radiation exposure: modelling basic mechanisms, Adv. Space Res. 31, 1557 (2003).

16. F. Ballarini, A. Ottolenghi, Models of chromosome aberration induction: an example based on radiation track structure, Cyt. Gen. Res. 104, 149 (2004).

17. F. Ballarini, A. Ottolenghi, A model of chromosome aberration induction: applications to space research, Radiat. Res. 164, 567 (2005).

18. F. Ballarini, A. Ottolenghi, A model of chromosome aberration induction and CML incidence at low doses, Radiat. Environ. Biophys. 43, 165 (2004).

19. K. George, M. Durante, V. Willingham, H. Wu, T. Yang, F.A. Cucinotta, Biological effectiveness of accelerated particles for the induction of chromosome damage measured in metaphase and interphase human lymphocytes, Radiat Res 160, 425 (2003). 